\section{Brucella canis in Commercial Dog Breeding Kennels, Ontario, Canada}

\author{
J. Scott Weese, Kathleen Hrinivich, \\ Maureen E.C. Anderson
}

University of Guelph, Guelph, Ontario, Canada (J.S. Weese);

Animal Hospital of Cambridge, Cambridge, Ontario (K. Hrinivich); Ministry of Agriculture, Food and Rural Affairs, Guelph

(M.E.C. Anderson)

DOI: https://doi.org/10.3201/eid2612.201144

We evaluated the prevalence of Brucella canis seropositivity in a convenience sample of dogs from commercial breeding kennels in Ontario, Canada. Overall, 127/1,080 $(11.8 \%)$ dogs from $23 / 63(37 \%)$ kennels were seropositive. The prevalence of positive dogs within kennels with $\geq 1$ positive dog ranged from $3.9 \%$ to $100 \%$ (median $33 \%$ ).

$B$ rucella canis is a dog-adapted Brucella species that most commonly causes reproductive disease and diskospondylitis in dogs and can be carried long-term and subclinically. Zoonotic infections are uncommonly reported (1-4), but may be underdiagnosed $(3,5)$.

In Canada, Brucella canis has been found predominantly in imported dogs. However, it was identified in 2 adult female dogs from a commercial breeding kennel in Ontario, Canada, in March 2019. We conducted an investigation of prevalence and distribution of B. canis in the broader commercial dog breeding population.

We collected serum samples from a convenience sample of dogs at commercial breeding kennels in southern Ontario, Canada. We used rapid slide agglutination test (RSAT) and followed up with positive results using 2-mercaptoethanol RSAT (2ME-RSAT). We performed PCR on whole (EDTA treated) blood from a subset of $B$. canis-seropositive dogs.

We identified positive RSAT and 2ME-RSAT tests in 127/1,080 (11.8\%) clinically normal dogs from 23/63 (37\%) kennels during March 15-December 18, 2019 (1-61 dogs/kennel, median 7). We considered reactive an additional $82(7.6 \%)$ dogs that were positive by RSAT but negative by 2ME-RSAT; 63 (77\%) of those were from kennels from which positive dogs were identified. The prevalence of positive dogs within kennels that had $\geq 1$ positive dog ranged from $3.9 \%$ to $100 \%$ (median $33 \%$ ). Whole blood samples from 20 dogs tested by PCR were all negative. We retested 130 dogs 4-6 weeks after the initial test (Table).

The seropositive rate contrasts with a 1980 study that reported $0.3 \%$ seroprevalence in dogs from south- western Ontario (6). Studies in other regions have reported seroprevalence rates of $0 \%-4.6 \%$; higher rates (e.g., 20\%-83\%) were reported in some breeding kennels (1,7-9). A structured approach to enrollment was not possible because enrollment was based on kennel operators' willingness to participate. Various population enrollment biases might be present in the prevalence estimate. These results should be taken as an indication of widespread presence of $B$. canis bacteria in this population, with high rates in some kennels and the potential for introduction of infected puppies into households.

Because B. canis infection is a notifiable disease in Ontario, we obtained data from 2013-2018 from the Ontario Ministry of Agriculture Food and Rural Affairs. Provincially, there were no positive test results for B. canis in dogs in 2013, 2015 and 2017, and 1-3 cases in each of 2014, 2016, and 2018. Because prior surveillance was limited, it is unclear whether this is a new problem or one that was previously overlooked. However, these 0-3 diagnoses/year and anecdotal data about recent reproductive disease in some affected kennels make it unlikely that $B$. canis infection was present but undiagnosed. The origin of the infection could not be properly investigated, but it was suspected to have originated from breeding dogs imported from eastern Europe in 2018.

Without a standard approach for clinical or surveillance testing for $B$. canis bacteria, we used the sensitive RSAT followed by the more specific 2MERSAT, which is considered a confirmatory test (10). Cases with RSAT-positive but 2ME-RSAT-negative results were common; most were subsequently negative. A possible cause was transient cross-reaction with Bordetella bronchiseptica vaccination or another pathogen; we could not investigate specifically because information about $B$. bronchiseptica vaccination or infection in these dogs was not available. The potential for false-positive results should be considered, particularly because infected dogs are often euthanized in accordance with regulatory requirements.

\begin{tabular}{lccc}
\hline $\begin{array}{l}\text { Table. Initial and follow-up serologic testing for Brucella canis in } \\
130 \text { dogs, Canada }\end{array}$ & \multicolumn{3}{l}{} \\
\hline Initial result & No. & Follow-up result* & No. (\%) \\
\hline Negative & 84 & Positive & $0(0)$ \\
& & Reactive & $0(0)$ \\
& & Negative & $84(100)$ \\
\hline Positive & 9 & Positive & $6(67)$ \\
& & Reactive & $1(11)$ \\
& & Negative & $2(22)$ \\
\hline Reactive & 37 & Positive & $1(0.3)$ \\
& & Reactive & $2(0.5)$ \\
& & Negative & $34(92)$ \\
\hline
\end{tabular}

*Follow-up testing was performed 4-6 weeks after the initial test. 
Limited PCR testing was performed because of negative results in the first 20 samples; negative results were presumed due to the intermittent nature of B. canis bacteremia in clinically normal animals. Although PCR or culture can provide a definitive diagnosis, sensitivity can be low for screening; it is higher when testing reproductive or fetal fluids or tissues from abortions or stillbirths for B. canis.

Limited clinical data were available. Some affected kennels reported substantial reproductive challenges presumably associated with brucellosis (e.g., small litter sizes, abortions, stillbirths, low conception rates) whereas no problems were reported in others. Whether this reflects lack of recognition of problems, subclinical infection, or early infection that had not yet resulted in overt reproductive disease is unclear.

Underdiagnosis of $B$. canis as a cause of nonspecific disease (e.g., undulating fever, fatigue, headache, malaise, chills, weight loss, hepatomegaly, splenomegaly, lymphadenopathy) and human brucellosis is a concern; physicians are more likely to perform serologic tests that target smooth Brucella species (B. abortus, $B$. suis, and $B$. melitensis). Physicians should consider the potential presence of $B$. canis in patients with disease suggestive of brucellosis, especially those with animal contact, and realize the limitations of serologic testing.

Brucella canis should be considered endemic to commercial dog kennels in Ontario, with potential human health risks. $B$. canis screening of breeding dogs is recommended (10), and testing of puppies from parents of unknown Brucella status is reasonable.

\section{Acknowledgments}

We thank Tim Pasma for providing B. canis reports from previous years in Ontario.

This study was supported by the Ontario Animal Health Network.

\section{About the Author}

Dr. Weese is a veterinary internist at the University of Guelph, Guelph, Ontario, Canada. His primary research interests are infectious diseases and infection control, and he has a particular interest in emerging diseases, zoonotic diseases, and antimicrobial stewardship.

\section{References}

1. Johnson CA, Carter TD, Dunn JR, Baer SR, Schalow MM, Bellay YM, et al. Investigation and characterization of Brucella canis infections in pet-quality dogs and associated human exposures during a 2007-2016 outbreak in Michigan. J Am Vet Med Assoc. 2018;253:15.

2. Lucero NE, Corazza R, Almuzara MN, Reynes E, Escobar GI, Boeri E, et al. Human Brucella canis outbreak linked to infection in dogs. Epidemiol Infect. 2010;138:280-5. https:/ / doi.org/10.1017/S0950268809990525

3. Lucero NE, Escobar GI, Ayala SM, Jacob N. Diagnosis of human brucellosis caused by Brucella canis. J Med Microbiol. 2005;54:457-61. https:// doi.org/10.1099/jmm.0.45927-0

4. Dentinger CM, Jacob K, Lee LV, Mendez HA, Chotikanatis $\mathrm{K}, \mathrm{McD}$ onough PL, et al. Human Brucella canis infection and subsequent laboratory exposures associated with a puppy, New York City, 2012. Zoonoses Public Health. 2015;62:40714. https://doi.org/10.1111/zph.12163

5. Hensel ME, Negron M, Arenas-Gamboa AM. Brucellosis in dogs and public health risk. Emerg Infect Dis. 2018;24:14016. https://doi.org/10.3201/eid2408.171171

6. Bosu WTK, Prescott JF. A serological survey of dogs for Brucella canis in southwestern Ontario. Can Vet J. 1980; 21:198-200.

7. Brower A, Okwumabua O, Massengill C, Muenks Q, Vanderloo P, Duster M, et al. Investigation of the spread of Brucella canis via the U.S. interstate dog trade. Int J Infect Dis. 2007;11:454-8. https://doi.org/10.1016/j.ijid.2006.12.009

8. Dahlbom M, Johnsson M, Myllys V, Taponen J, Andersson M. Seroprevalence of canine herpesvirus- 1 and Brucella canis in Finnish breeding kennels with and without reproductive problems. Reprod Domest Anim. 2009;44:128-31. https://doi.org/10.1111/j.1439-0531.2007.01008.x

9. Higgins R, Hoquet F, Bourque R, Gosselin Y. A serological survey for Brucella canis in dogs in the Province of Quebec. Can Vet J. 1979;20:315-7.

10. Bramlage DJ, Fortney W, Kesler RM, Mabray CJ, Mason JW, Reinhold $\mathrm{H}$, et al. Best practices for Brucella canis prevention and control in dog breeding facilities. US Department of Agriculture. 2015 [cited 2020 Oct 21]. https:// www.aphis. usda.gov/animal_welfare/downloads/brucella_canis_ prevention.pdf

Address for correspondence: J. Scott Weese, Centre for Public Health and Zoonoses, Ontario Veterinary College, University of Guelph, Guelph, ON, N1G2W1 Canada; email: jsweese@ovc.uoguelph.ca 